\title{
ELK1-induced upregulation of long non-coding TNK2-AS1 promotes the progression of acute myeloid leukemia by EZH2-mediated epigenetic silencing of CELF2
}

Dongfang Guo

Zhumadian Central Hospital

Airong Zhang ( $\sim$ airongz@21cn.com )

Zhumadian Central Hospital https://orcid.org/0000-0002-9613-1755

Meifang Suo

Zhumadian Central Hospital

Ping Wang

Zhumadian Central Hospital

Yile Liang

The First Affiliated Hospital of Henan University of Science and Technology

\section{Research Article}

Keywords: LncRNA TNK2-AS1, ELK1, EZH2, CELF2, acute myeloid leukemia

Posted Date: August 13th, 2021

DOI: https://doi.org/10.21203/rs.3.rs-792325/v1

License: (c) (1) This work is licensed under a Creative Commons Attribution 4.0 International License.

Read Full License 


\section{Abstract}

Acute myeloid leukemia (AML) is the commonest hematological malignancy in the world. Long noncoding RNAs (LncRNAs) have been suggested as key regulators of cancer development and progression in AML. As a member of IncRNA family, the biological role and mechanisms of tyrosine kinase non receptor 2 antisense RNA 1 (TNK2-AS1) in AML is still unclear. The expression of TNK2-AS1 was measured by using RT-qPCR in AML cell lines. The changes in TNK2-AS1 shRNA-transfected HL-60 and THP-1 cell proliferation, apoptosis and differentiation were detected by using CCK-8, EdU, flow cytometry, Western blot and NBT assays. Molecular control of TNK2-AS1 on CUGBP Elav-like family member 2 (CELF2) and ETS domain-containing protein-1 (ELK1) on TNK2-AS1 was assessed by chromatin immunoprecipitation (ChIP), RT-qPCR, Western blot and RNA immunoprecipitation (RIP) assays. TNK2AS1 expression was upregulated in AML cell lines and negatively correlated with survival patients. Knockdown of TNK2-AS1 markedly reduced AML cell proliferation and promoted apoptosis and differentiation. Likewise, TNK2-AS1 knockdown significantly suppressed tumor growth in vivo. Mechanistically, the upregulation of TNK2-AS1 was activated by transcription factor ELK1. Beside, we uncovered that TNK2-AS1 exerted tumor-promoting effect through silencing CELF2 via binding with EZH2, thus activating PI3K/Akt pathway in AML cells. Elevated expression of TNK2-AS1 was induced by ELK1 and facilitated AML progression by suppressing CELF2 expression via EZH2-mediated epigenetic silencing, suggesting TNK2-AS1 may be a promising therapeutic target and prognostic marker for AML patients.

\section{Introduction}

The most commonly detected type of acute leukemia, accounting for highest rate of mortality worldwide, is acute myeloid leukemia (AML) [1]. AML is characterized by impaired interference and apoptosis, uncontrolled malignant proliferation and blocked differentiation in leukemic cells [2,3]. Among all kinds of hematological malignancies, AML is the most difficult to treat, and the 5 -year survival rate is only $25 \%$ $[4,5]$. The current treatment of leukemia mainly includes chemotherapy, radiotherapy and bone marrow transplantation, but the prognosis of many patients is still very poor [6]. Therefore, an in-depth knowledge of the molecular mechanism of the malignant biological behaviors of AML may provide novel and effective therapy of AML.

Long non-coding RNAs (LncRNAs) are defined as a class of RNA molecules with length over 200 nucleotides. LncRNAs participate in diverse cancers, such as non-small cell lung cancer, cervical cancer and AML [7-9]. Growing evidence has demonstrated that IncRNAs can function as oncogenes or tumor suppressors to affect various biological processes including epigenetic regulation, transcriptional regulation and post transcriptional regulation $[10,11]$. Moreover, dysregulation of IncRNAs have been identified to involve in multiple cellular processes such as metastasis, differentiation and apoptosis [12, 13]. For example, IncRNA HOXA-AS2 acts as an oncogene in AML by interacting with EZH2 to inhibiting LATS2 expression [3]. LINC00963 acts as a ceRNA for miR-608 to regulate the expression of MMP-15, thus facilitating AML development [9]. LncRNA CCAT1 is highly expressed in AML and could suppress 
cell differentiation and promote cell growth by regulating miR-155 [14]. These findings suggest that IncRNAs play an important role in the pathogenesis of $A M L$ and potential novel biomarkers for $A M L$ diagnosis and therapy.

TNK2-AS1 was initially found to be upregulated in non-small cell lung cancer (NSCLC) tissues [15]. Cai et al. uncovered that TNK2-AS1 promoted proliferation and migration of human aortic smooth muscle cells (HASMCs) through regulating VEGFA and FGF1 by sponging miR-150-5p [16]. In osteosarcoma (OS), TNK2-AS1 knockdown suppressed WDR1 expression through competitively binding miR-4319, inhibiting OS cell proliferation, invasion and inducing apoptosis [17]. However, the expression and role of TNK2-AS1 in AML have not been characterized. In current study, the expression of TNK2-AS1 was firstly verified and we revealed that TNK2-AS1 was powerfully expressed in AML cells. Unsurprisingly, TNK2-AS1 knockdown suppressed AML cell proliferation, induced apoptosis and differentiation. More importantly, we found that ELK1 directly activated TNK2-AS1 transcription, and TNK2-AS1 could bind to EZH2 to downregulate CELF2 expression and activate PI3K/Akt signaling pathway in AML.

\section{Materials And Methods}

\section{Cell lines and culture}

HS-5 and AML cell lines ((HEL, NB4, HL-60, and THP-1) were purchased from the Cell Bank of the Chinese Academy of Sciences (Shanghai, China). All cells were cultured in RPMI-1640 medium (Invitrogen) containing $10 \%$ fetal bovine serum (FBS) and incubated in a humidified atmosphere containing $5 \% \mathrm{CO}_{2}$ at $37^{\circ} \mathrm{C}$.

\section{Cell Transfection}

To overexpress the expression of TNK2-AS1, the full sequence of TNK2-AS1 was introduced into the pcDNA3.1 vector (Invitrogen, Carlsbad, CA, USA) to generate pcDNA-TNK2-AS1 constructs. Likewise, to silence the expression of TNK2-AS1, ELK1, EZH2 and CELF2, shRNAs specifically targeting them were synthesized and obtained from Genepharma (Shanghai, China). The above negative controls (vector and sh-NC) were also synthesized by Genepharma (Shanghai, China). Lipofectamine 2000 from Invitrogen was utilized to transfect above in cells.

\section{Quantitative real-time PCR (RT-qPCR)}

Total RNAs were extracted by using Trizol (Invirtogen, Carlsbad, CA, USA). The cDNA was synthesized from total RNA by using Prime Script RT reagent (TaKaRa, Tokyo, Japan). Quantitative real time-PCR (RTqPCR) was performed with SYBR Taq by using an Agilent Bioanalyzer 2100 (Agilent Technologies, Santa Clara, CA, US). Briefly, reactions were incubated at $95^{\circ} \mathrm{C}$ for $10 \mathrm{~s}$ for an initial denaturation, followed by 40 cycles at $95^{\circ} \mathrm{C}$ for $15 \mathrm{~s}$ and then $60^{\circ} \mathrm{C}$ for $1 \mathrm{~min}$. All reactions were run in triplicate, and the relative expression of mRNA or miRNA was calculated using the $2^{-\triangle \Delta C T}$ method. Human $U 6$ as an endogenous 
control. The relative gene expression was normalized to glyceraldehyde 3-phosphate dehydrogenase (GAPDH).

\section{Cell Counting Kit-8 (CCK-8)}

HL-60 and THP-1 cells were seeded onto 6-well plates with six replicate wells and then transfected with TNK2-AS1 shRNA, CELF2 shRNA or sh-NC. After $48 \mathrm{~h}$ transfection, $\mathrm{HL}-60$ and THP-1 cells were plated into 96 -well plates and incubated for $12 \mathrm{~h}$ in a humidified incubator containing $5 \% \mathrm{CO}_{2}$ at $37^{\circ} \mathrm{C}$ for 24, 48, 72 and $96 \mathrm{~h}$. Then, HL-60 and THP-1 cells were treated with CCK-8 solution (Invitrogen) for $4 \mathrm{~h}$. The absorbance was determined at the wavelength of $450 \mathrm{~nm}$ by using microplate reader (Molecular devices, Shanghai, China).

\section{EdU assays}

After transfection for $24 \mathrm{~h}$, cell media was replaced with fresh media supplemented with $50 \mu \mathrm{m}$ EdU reagent for $2 \mathrm{~h}$ followed by fixation with $4 \%$ phosphate-buffered paraformaldehyde. Then the cells were stained with $100 \mu \mathrm{L}$ of AdoLo and Hoechst 33 342, respectively. The number of EdU-positive cells was analyzed by fluorescence microscopy (Olympus BX51). The EdU-positive ratio was calculated as the ratio of the number of EdU-positive cells to the total Hoechst 33 342-labeled cells (blue cells).

\section{Flow cytometry analysis}

HL-60 and THP-1 cells after transfected were cultured for $48 \mathrm{~h}$, and then digested with trypsin. After centrifugation, cells were harvested and re-suspended with binding buffer. Then, cells were stained with Annexin V-fluorescein isothiocyanate (V-FITC) and Propidium iodide (PI) for $15 \mathrm{~min}$ in dark. Fluorescence signals were analyzed directly by flow cytometry using the Cell Quest program (Becton Dickinson, Franklin, NJ).

\section{Nitroblue tetrazolium (NBT) assay}

The transfected cells were inoculated in a six-well plate and were collected. A $10 \mu$ l aliquot of NBT solution composed of $10 \mathrm{mg} / \mathrm{ml} \mathrm{NBT}$ (Sigma-Aldrich) and $2 \mu \mathrm{g} / \mathrm{ml}$ PMA (Sigma-Aldrich), was added to each well, and then cells were incubated for $30 \mathrm{~min}$ at $37^{\circ} \mathrm{C}$. Then, light microscopy was used for analysis. The positive cells ratio was analyzed by light microscopy

\section{Western blot analysis}

Cells were collected and lysed with RIPA lysis buffer (Beyotime, Shanghai, China) for the Western blot analysis. Proteins in the lysates ( $20 \mu \mathrm{g}$ per lane) were separated by $10 \%$ sodium dodecyl sulfatepolyacrylamide gel electrophoresis (SDS-PAGE) and transferred onto polyvinylidene fluoride (PVDF) membranes. After blocking with $5 \%$ non-fat milk, the membranes were incubated with primary antibodies against EZH2 (1:1000, ab191250, Abcam), CELF2 (1:1000, ab186430, Abcam), ELK1 (1:1000, ab32106, Abcam), CD11b (1:1000, ab13357, Abcam) and CD14 (1:1000, ab182032, Abcam) overnight at room 
temperature, and then incubated with horseradish peroxidase (HRP)-labeled goat anti-mouse secondary antibody at room temperature for $2 \mathrm{~h}$. The protein bands were visualized using the enhanced chemiluminescence reagents (Millipore, MA, USA). The expression of relative protein was obtained by the gray value ratio of the target protein to the internal reference GAPDH.

\section{Chromatin immunoprecipitation (ChIP)}

ChIP assay was performed with the EZ-ChIP Kit (Millipore). Formaldehyde was used to incubate with cells to produce DNA-protein crosslinks. Cross-linked chromatin was sonicated into 200 to 500-base pairs fragments and then the cell lysates were separately incubated with antibodies against ELK1, EZH2 and H3K27me3 (Millipore). The precipitated chromatin DNA were determined by RT-qPCR.

\section{RNA immunoprecipitation (RIP)}

RIP assay was performed in HL-60 cells by using a Magna RIP RNA-Binding Protein Immunoprecipitation kit (Millipore, Billerica, MA, USA). The negative control, normal IgG and antibodies against EZH2 were bond with magnetic beads and cultured with the cell lysates in RIP buffer. The co-precipitated RNAs were detected and quantified by RT-qPCR assay.

\section{Luciferase reporter assay}

Cells were plated in 96-well plates and transfected with a compound of $5 \mathrm{ng}$ pRL-CMV Renilla luciferase reporter and $50 \mathrm{ng}$ firefly luciferase reporter. At last, the luciferase activity was measured with the dual luciferase reporter assay system (Promega Corporation, Madison, WI, USA).

\section{Tumor xenografts}

All animal care and experiments were carried out in accordance with the guidance of the National Institutes of Health and approved by the Ethics Committee of Zhumadian Central Hospital. We selected 4week-old NCG mice with severe immunodeficiency for tumor xenotransplantation experiments to study the effect of TNK2-AS1 on tumor proliferation and differentiation. In tumor growth assay in vivo, THP-1 cells stably transfected with TNK2-AS1 sh-RNA (LV-sh-TNK2-AS1) and respective negative control (LV-shNC) were subcutaneously injected into the upper back of the nude mice $\left(1 \times 10^{7}, 200 \mu \mathrm{L}\right)$. Tumor length and width were recorded weekly to calculate the tumor volume according to the formula: volume=length $x$ width $^{2} / 2$. After 5 weeks, the tumor was removed for weight measurement, RT-qPCR and Western blot analysis.

\section{Statistical Analysis}

All statistical computations were performed by using statistical product and service solutions (SPSS) 23 software (SPSS Inc., Chicago, IL, USA). Data were expressed as mean \pm standard deviation. Student's ttest was used to analyze the differential expression. $P<0.05$ suggested that the difference was statistically significant. 


\section{Results}

\section{TNK2-AS1 levels were increased in AML}

In order to investigate the biological role of TNK2-AS1 in AML development, we first performed GEPIA database to assess the expression of TNK2-AS1 in AML patients. The results showed that the expression of TNK2-AS1 was remarkably upregulated in AML patients $(n=173)$ compared with healthy human individuals $(n=70)$ (Fig. 1A). Furthermore, analysis of GEPIA database showed that the expression of TNK2-AS1 was negatively correlated with the overall survival of AML patients (Fig. 1B). To further confirmed the results of above analysis, we examined the expression of TNK2-AS1 in AML cell lines by RT-qPCR. As expected, the results showed that TNK2-AS1 was highly expressed in leukemia cell lines (HEL, NB4, HL-60 and THP-1) compared with normal HS-5 cells (Fig. 1C). Taken together, these results revealed that TNK2-AS1 may play an oncogene role in AML.

\section{Knockdown of TNK2-AS1 inhibited proliferation, induced differentiation and apoptosis of AML cells}

To further explore whether TNK2-AS1 exerted oncogenic function in AML, we transfected TNK2-AS1 shRNA or negative control (sh-NC) into HL-60 and THP-1 cells. The results of RT-qPCR assay showed that TNK2-AS1 shRNA had a significantly inhibitory effect on TNK2-AS1 expression in both cells (Fig. 2A). CCK-8 analysis revealed that silencing of TNK2-AS1 suppressed proliferation of both HL-60 and THP-1 cells (Fig. 2B). EdU assay showed that cell proliferation was notably decreased in TNK2-AS1-depleted HL60 and THP-1 cells than that in control (Fig. 2C). Moreover, flow cytometry assay indicated that knockdown of TNK2-AS1 induced an increase in the percentage of apoptotic of HL-60 and THP-1 cells (Fig. 2D). We then detected the expression of classic differentiation markers of leukemia in HL-60 and THP-1 cells after TNK2-AS1 knockdown. The results of RT-qPCR and western blotting showed that the protein levels of CD11b and CD14 were increased in sh-TNK2-AS1 transfected group compared to sh-NC group (Fig. 2E). Subsequently, nitroblue tetrazolium (NBT) reduction showed that TNK2-AS1 knockdown remarkably induced AML cell differentiation compared with the sh-NC group (Fig. 2F).

\section{TNK2-AS1 was upregulated by the transcription factor ELK1 in AML cells}

It was spot from online database JASPAR that the potential binding sites for ELK1 in the promoter region of TNK2-AS1 were found, suggesting that ELK1 may be a transcription factor for TNK2-AS1 (Fig. 3A). Then, we found that ELK1 was upregulated in AML cell lines (Fig. 3B). To confirm the relationship between them, ChIP assay was conducted and revealed that ELK1 could directly bind to the promoter region of TNK2-AS1 in HL-60 and THP-1 cells (Fig. 3C). Afterward, luciferase reporter vectors containing serial truncations of TNK2-AS1 promoter were constructed and transfected into HEK-293T cells. The results showed that the luciferase activity of site 1 reporter was strengthened by ELK1 upregulation in HL60 and THP-1 cells, whereas had no effect on site 2 reporter (Fig. 3D). Besides, to further identify the interaction between TNK2-AS1 and ELK1, we transfected ELK1 overexpression plasmid and shRNA into HL-60 and THP-1 cells. RT-qPCR results showed that the expression of ELK1 mRNA was elevated in ELK1 overexpressed group and declined in ELK1 knockdown group (Fig. 3E). On the contrary, the 
expression of TNK2-AS1 was significantly reduced by ELK1 overexpression and remarkably elevated by TNK2-AS1 knockdown in HL-60 and THP-1 cells (Fig. 3F).

\section{TNK2-AS1 interacted with EZH2 to suppress CELF2 expression}

To investigate the potential mechanism for TNK2-AS1 in AML, we first assessed the distribution of TNK2AS1 in HL-60 and THP-1 cells and uncovered that TNK2-AS1 expression was mainly located in the nucleus (Fig. 4A), implying that TNK2-AS1 may be involved in transcriptional regulation. To investigate whether TNK2-AS1 was physically associated with EZH2 that regulated target genes at transcriptional level in the nucleus, we carried out a RIP assay. The results revealed that there is a strong physical interaction between TNK2-AS1 and EZH2 (Fig. 4B). EZH2 is a histone methylation regulator mainly distributed in the nucleus, which can target a variety of tumor suppressor genes to promote tumor development, including CELF2. We further uncovered that silencing of TNK2-AS1 significantly elevated the protein level of CELF2 in HL-60 cells (Fig. 4C). Likewise, knockdown of EZH2 remarkably repressed the protein level of EZH2 (Fig. 4D), whereas markedly increased the protein level of CELF2 in HL-60 cells (Fig. 4E). Moreover, ChIP assay was used to explore whether TNK2-AS1 knockdown inhibited the expression of CELF2 by EZH2-meidated epigenetic silencing. The results manifested that TNK2-AS1 knockdown restrained EZH2 binding to the promoter region of CELF2 gene and EZH2-mediated histone $\mathrm{H} 3$ lysine 27 trimethylation (H3K27me3) modification in HL-60 cells (Fig. 4F).

\section{Knockdown of CELF2 involved in TNK2-AS1-mediated oncogenic function in AML}

To explore whether CELF2 was implicated in the TNK2-AS1-mediated AML progression, the TNK2-AS1 shRNA was transfected alone or together with CELF2 shRNA into HL-60 and THP-1 cells. RT-qPCR analysis affirmed that the upregulation of CELF2 by TNK2-AS1 knockdown was reversed by CELF2 shRNA (Fig. 5A). CCK-8 and EdU assays proofed that the suppressed cell proliferative ability in sh-TNK2AS1-transfected was reversed by CELF2 knockdown (Fig. 5B and C). Fow cytometry assay demonstrated that the decrease CELF2 expression could rescue TNK2-AS1 deficiency-induced cell apoptosis (Fig. 5D). In addition, it was ascertained by western blot and RT-qPCR assays that silencing of CELF2 could attenuate the promoting effect of TNK2-AS1 knockdown on CD11b and CD14 expression and the inhibitory effect of TNK2-AS1 knockdown on PI3K and Akt phosphorylation (Fig. 5E and F).

\section{Knockdown of TNK2-AS1 suppressed AML tumorigenesis in vivo}

To further determine the carcinogenic effect of TNK2-AS1 in AML in vivo, we next injected nude mice with THP-1 cells that were transfected with lentiviruses carrying sh-TNK2-AS1 or sh-NC. From tumor growth curve, we found that the tumor volume of TNK2-AS1 knockdown group was significantly inhibited compared with the sh-NC group (Fig. 6A). Simultaneously, tumor weight of TNK2-AS1 knockdown group was remarkably lower than that of the sh-NC group (Fig. 7B). Furthermore, RT-qPCR analysis showed that TNK2-AS1 expression in tumors was downregulated in TNK2-AS1 knockdown group compared with shNC group (Fig. 6C). In addition, western blot showed that TNK2-AS1 knockdown upregulated CELF2 
protein and suppressed PI3K and Akt phosphorylation (Fig. 6D and E). Collectively, the above results suggested that TNK2-AS1 may function as an oncogenic role in AML.

\section{Discussion}

LncRNAs have been reported to be potential diagnostic and prognostic markers for multiple cancers including AML $[14,18,19]$. Increasing evidence has been demonstrated that IncRNAs are key players in AML progression [20-22]. In current study, we firstly elucidated the biological role of TNK2-AS1 in AML. At first, we investigated the expression of TNK2-AS1 in AML. The predicted results from the database website (GEPIA) showed that the expression of TNK2-AS1 was highly expressed in AML patients, and was also negatively related to the survival of AML patients. We further found that TNK2-AS1 was noticeably upregulated in AML cell lines, which was consistent with previous studies showing that TNK2AS1 expression was increased in NSCLC [15], atherosclerosis [16] and OS [17]. We continue to detect the upstream transcription factor of TNK2-AS1 by bioinformatic analysis and experiments, and uncovered ELK1 could directly bind to TNK2-AS1 promoter region to transcriptionally activate it. Accumulated evidence indicated that ELK1 was an important transcription activator of IncRNA [23-25]. Most importantly, the results of rescue assays indicated that knockdown of TNK2-AS1 significantly suppressed proliferation and induced apoptosis and differentiation of AML cells in vitro, and inhibited tumor growth in vivo.

It has been proofed by extesnsive researched that IncRNAs regulated cancer related biological processed through a variety of different molecular mechanisms including binding to RNA-binding proteins (RBPs). For instance, TNK2-AS1 facilitated angiogenesis by interacting with STAT3 to elevating VEGFA expression [15]. Gao et al. found that HOTAIR facilitated leukemogenesis by EZH2-mediated epigenetic silencing of p15 [26]. HOXA-AS2 exerted carcinogenic effect through binding with EZH2 and reducing LATS2 expression in AML [3]. It has been identified that EZH2 is a highly conserved histone methyltransferase, which can restrain the expression of downstream tumor suppressor genes by triggering $\mathrm{H} 3 \mathrm{~K} 27$ me3 trimethylation, thus affecting cellular fate $[7,27]$. A large number of IncRNAs bind with EZH2 to regulating the expression of target genes such as PTEN [7], p15 [26] and KLF2 [28]. Our study revealed that TNK2-AS1 inhibited CELF2 expression via the recruitment of EZH2 to facilitate AML cell proliferation and reduce apoptosis and differentiation.

CELF2 is recognized as an RBPs and has been reported as a tumor inhibitor in various cancers $[29,30]$. Dysregulation of CELF2 could regulate cell cycle, proliferation, migration, invasion and apoptosis [31, 32]. In hepatocellular carcinoma, IncRNA CRNDE promoted epigenetic suppression of CELF2 by binding to EZH2 to facilitate proliferation, migration and chemoresistance [29]. Wu et al. showed that STYXL1 accelerated the development of hepatocellular carcinoma via inhibiting CELF2 expression through the $\mathrm{PI3K} /$ Akt pathway [33]. Yeung et al. illustrated that CELF2 play an important role in the PI3K/Akt pathway [34]. Activation of PI3K/Akt pathway could promote the occurrence of AML [22, 35]. Herein, we uncovered that silencing of TNK2-AS1 could increase CELF2 expression level in AML cells and EZH2 could bind to 
CELF2 promoter region. Afterward, rescue assays identified that CELF2 and PI3K/Akt pathway were implicated in the TNK2-AS1-mediated AML progression.

\section{Conclusions}

In conclusion, our results revealed that TNK2-AS1 was remarkably upregulated in AML patients and partly activated by ELK1. Knockdown of TNK2-AS1 noticeably restrained AML cell proliferation while induced apoptosis and differentiation. In addition, TNK2-AS1 exerted oncogenic role in AML through regulation of the EZH2/CELF2/PI3K/Akt pathway. These findings provided novel potential diagnostic or therapeutic target for AML.

\section{Declarations}

\section{Acknowledgements}

The authors thank Zhumadian Central Hospital for for its support.

\section{Author contributions}

Dongfang Guo and Airong Zhang contributed to the experiment design, manuscript draft, and data analysis. Meifang Suo contributed to the experiment implementation, manuscript draft, and data analysis. Ping Wang performed the experiment. Yile Liang analyzed the data. All authors read and approved the final manuscript.

\section{Availability of data and materials}

The datasets used during the present study are available from the corresponding author upon reasonable request.

\section{Ethics approval and consent to participate}

The animal use and care was approved by the Committee for Ethical Review of Research of the Zhumadian Central Hospital and all animal studies were performed according to the guidelines for the ethical review of laboratory animal welfare National Standard GB/T 35,892-2018 issued by the People's Republic of China. The in vivo animal study was also carried out in compliance with the ARRIVE guidelines 2.0.

\section{Conflicts of Interest}

The authors declare no competing interests

\section{References}


1. O'Donnell MR, Tallman MS, Abboud CN, et al. Acute myeloid leukemia, version 2.2013. Journal of the National Comprehensive Cancer Network: JNCCN. 2013;11(9):1047-55. https://doi.org/10.6004/jnccn.2013.0127.

2. Peters $\mathrm{AH}$, Schwaller J. Epigenetic mechanisms in acute myeloid leukemia. Progress in drug research. Fortschritte der Arzneimittelforschung. Progres des recherches pharmaceutiques, 2011, 67: 197-219. https://doi.org/10.1007/978-3-7643-8989-5_10.

3. Feng Y, Hu S, Li L, et al. Long noncoding RNA HOXA-AS2 functions as an oncogene by binding to EZH2 and suppressing LATS2 in acute myeloid leukemia (AML). Cell death disease. 2020;11(12):1025. https://doi.org/10.1038/s41419-020-03193-3.

4. ]4], Deschler B and Lübbert M. Acute myeloid leukemia: epidemiology and etiology. Cancer, 2006, 107(9): 2099 - 107. https://doi.org/10.1002/cncr.22233.

5. Bullinger $L$, Ehrich $M$, Döhner K, et al. Quantitative DNA methylation predicts survival in adult acute myeloid leukemia. Blood, 2010, 115(3): 636 - 42. https://doi.org/10.1182/blood-2009-03-211003.

6. Estey EH. Acute myeloid leukemia: 2012 update on diagnosis, risk stratification, and management. Am J Hematol. 2012;87(1):89-99. https://doi.org/10.1002/ajh.22246.

7. Zang X, Gu J, Zhang J, et al. Exosome-transmitted IncRNA UFC1 promotes non-small-cell lung cancer progression by EZH2-mediated epigenetic silencing of PTEN expression. Cell death disease. 2020;11(4):215. https://doi.org/10.1038/s41419-020-2409-0.

8. Rui $X, X u Y$, Jiang $X$, et al. Long non-coding RNA C5orf66-AS1 promotes cell proliferation in cervical cancer by targeting miR-637/RING1 axis. Cell death disease. 2018;9(12):1175. https://doi.org/10.1038/s41419-018-1228-z.

9. Zuo W, Zhou K, Deng M, et al. LINC00963 facilitates acute myeloid leukemia development by modulating miR-608/MMP-15. Aging. 2020;12(19):18970-81. https://doi.org/10.18632/aging.103252.

10. Han Li C, Chen Y. Small and Long Non-Coding RNAs: Novel Targets in Perspective Cancer Therapy. Curr Genom. 2015;16(5):319-26. https://doi.org/10.2174/1389202916666150707155851.

11. Yang G, Lu X, Yuan L. LncRNA: a link between RNA and cancer. Biochimica et biophysica acta, 2014, 1839(11): 1097-109. https://doi.org/10.1016/j.bbagrm.2014.08.012.

12. Xiong WC, Han N, Wu N, et al. Interplay between long noncoding RNA ZEB1-AS1 and miR-101/ZEB1 axis regulates proliferation and migration of colorectal cancer cells. American journal of translational research. 2018;10(2):605-17.

13. Pan $\mathrm{Y}, \mathrm{Wu} \mathrm{Y}, \mathrm{Hu} \mathrm{J}$, et al. Long noncoding RNA HOTAIR promotes renal cell carcinoma malignancy through alpha-2, 8-sialyltransferase 4 by sponging microRNA-124. Cell proliferation. 2018;51(6):e12507. https://doi.org/10.1111/cpr.12507.

14. Chen L, Wang W, Cao L, et al. Long Non-Coding RNA CCAT1 Acts as a Competing Endogenous RNA to Regulate Cell Growth and Differentiation in Acute Myeloid Leukemia. Mol Cells. 2016;39(4):330-6. https://doi.org/10.14348/molcells.2016.2308. 
15. Wang $Y$, Han D, Pan L, et al. The positive feedback between IncRNA TNK2-AS1 and STAT3 enhances angiogenesis in non-small cell lung cancer. Biochem Biophys Res Commun. 2018;507(1-4):185-92. https://doi.org/10.1016/j.bbrc.2018.11.004.

16. Cai T, Cui X, Zhang K, et al. LncRNA TNK2-AS1 regulated ox-LDL-stimulated HASMC proliferation and migration via modulating VEGFA and FGF1 expression by sponging miR-150-5p. J Cell Mol Med. 2019;23(11):7289-98. https://doi.org/10.1111/jcmm.14575.

17. Yao W, Yan Q, Du X, et al. TNK2-AS1 upregulated by YY1 boosts the course of osteosarcoma through targeting miR-4319/WDR1. Cancer Sci. 2020. https://doi.org/10.1111/cas.14727.

18. Shang AQ, Wang WW, Yang YB, et al. Knockdown of long noncoding RNA PVT1 suppresses cell proliferation and invasion of colorectal cancer via upregulation of microRNA-214-3p. Am J Physiol Gastrointest Liver Physiol. 2019;317(2):G222-g232. https://doi.org/10.1152/ajpgi.00357.2018.

19. Tian Y, Ma R, Sun Y, et al. SP1-activated long noncoding RNA IncRNA GCMA functions as a competing endogenous RNA to promote tumor metastasis by sponging miR-124 and miR-34a in gastric cancer. Oncogene. 2020;39(25):4854-68. https://doi.org/10.1038/s41388-020-1330-4.

20. Luo H, Zhu G, Xu J, et al. HOTTIP IncRNA Promotes Hematopoietic Stem Cell Self-Renewal Leading to AML-like Disease in Mice. Cancer cell. 2019;36(6):645-59.e8. https://doi.org/10.1016/j.ccell.2019.10.011.

21. Fernando TR, Contreras JR, Zampini M, et al. The IncRNA CASC15 regulates SOX4 expression in RUNX1-rearranged acute leukemia. Mol Cancer. 2017;16(1):126. https://doi.org/10.1186/s12943017-0692-x.

22. Yang Y, Dai W, Sun Y, et al. Long non-coding RNA linc00239 promotes malignant behaviors and chemoresistance against doxorubicin partially via activation of the PI3K/Akt/mTOR pathway in acute myeloid leukaemia cells. Oncol Rep. 2019;41(4):2311-20. https://doi.org/10.3892/or.2019.6991.

23. Sheng K, Lu J, Zhao H. ELK1-induced upregulation of IncRNA HOXA10-AS promotes lung adenocarcinoma progression by increasing $W n t / \beta$-catenin signaling. Biochem Biophys Res Commun. 2018;501(3):612-8. https://doi.org/10.1016/j.bbrc.2018.04.224.

24. Su X, Teng J, Jin G. Biomedicine. et al. ELK1-induced upregulation of long non-coding RNA MIR100HG predicts poor prognosis and promotes the progression of osteosarcoma by epigenetically silencing LATS1 and LATS2. \& pharmacotherapy = Biomedecine pharmacotherapie. 2019;109:78897. https://doi.org/10.1016/j.biopha.2018.10.029.

25. Wang W, Chen S, Song X, et al. ELK1/IncRNA-SNHG7/miR-2682-5p feedback loop enhances bladder cancer cell growth. Life sciences. 2020;262:118386. https://doi.org/10.1016/j.lfs.2020.118386.

26. Gao S, Zhou B, Li H, et al. Long noncoding RNA HOTAIR promotes the self-renewal of leukemia stem cells through epigenetic silencing of p15. Experimental hematology. 2018;67:32-40.e3. https://doi.org/10.1016/j.exphem.2018.08.005.

27. Sauvageau M, Sauvageau G. Polycomb group proteins: multi-faceted regulators of somatic stem cells and cancer. Cell stem cell. 2010;7(3):299-313. https://doi.org/10.1016/j.stem.2010.08.002. 
28. Bai Z, Wu Y, Bai S, et al. Long non-coding RNA SNGH7 Is activated by SP1 and exerts oncogenic properties by interacting with EZH2 in ovarian cancer. J Cell Mol Med. 2020;24(13):7479-89. https://doi.org/10.1111/jcmm.15373.

29. Xie SC, Zhang JQ, Jiang XL, et al. LncRNA CRNDE facilitates epigenetic suppression of CELF2 and LATS2 to promote proliferation, migration and chemoresistance in hepatocellular carcinoma. Cell death disease. 2020;11(8):676. https://doi.org/10.1038/s41419-020-02853-8.

30. Ramalingam S, Ramamoorthy P, Subramaniam D, et al. Reduced Expression of RNA Binding Protein CELF2, a Putative Tumor Suppressor Gene in Colon Cancer. Immuno-gastroenterology. 2012;1 (1):2733. https://doi.org/10.7178/ig.1.1.7.

31. Guo Q, Wu Y, Guo X, et al. The RNA-Binding Protein CELF2 Inhibits Ovarian Cancer Progression by Stabilizing FAM198B. Molecular therapy. Nucleic acids. 2021;23:169-84. https://doi.org/10.1016/j.omtn.2020.10.011.

32. Wang J, Liu L, Sun Y, et al. miR-615-3p promotes proliferation and migration and inhibits apoptosis through its potential target CELF2 in gastric cancer. Biomedicine pharmacotherapy = Biomedecine pharmacotherapie. 2018;101:406-13. https://doi.org/10.1016/j.biopha.2018.02.104.

33. Wu JZ, Jiang N, Lin JM, et al. STYXL1 promotes malignant progression of hepatocellular carcinoma via downregulating CELF2 through the PI3K/Akt pathway. Eur Rev Med Pharmacol Sci. 2020;24(6):2977-85. https://doi.org/10.26355/eurrev_202003_20662.

34. Yeung YT, Fan S, Lu B, et al. CELF2 suppresses non-small cell lung carcinoma growth by inhibiting the PREX2-PTEN interaction. Carcinogenesis. 2020;41(3):377-89. https://doi.org/10.1093/carcin/bgz113.

35. Zhou JD, Li XX, Zhang TJ, et al. MicroRNA-335/ID4 dysregulation predicts clinical outcome and facilitates leukemogenesis by activating PI3K/Akt signaling pathway in acute myeloid leukemia. Aging. 2019;11(10):3376-91. https://doi.org/10.18632/aging.101991.

\section{Figures}

A

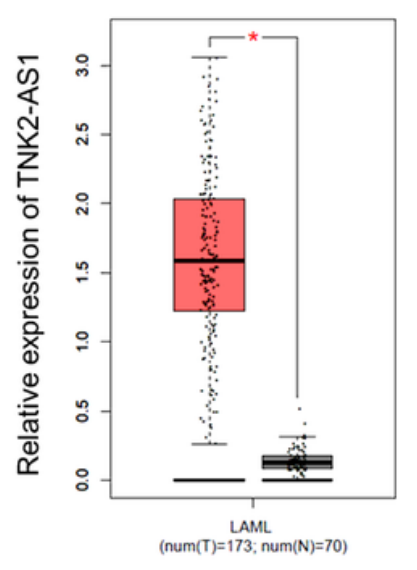

B

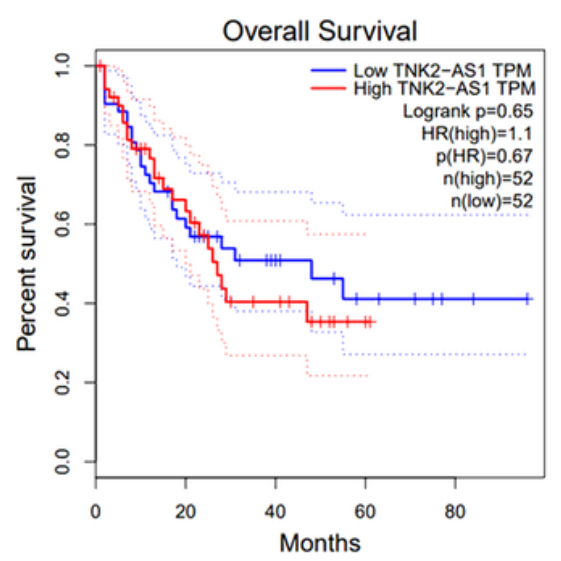

C

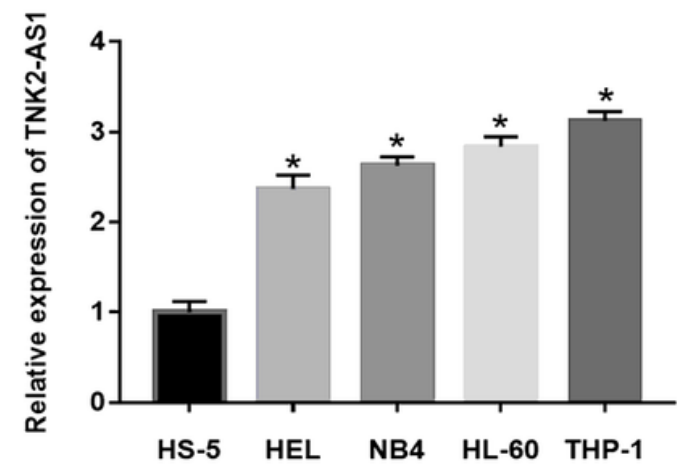


Figure 1

Relative expression of TNK2-AS1 in AML tissues and cell lines. (A) Box plots for TNK2-AS1 gene expression in AML and normal tissues from GEPIA. (B) Correlation between TNK2-AS1 expression and overall survival were detected in GEPIA. (C) The expression of TNK22-AS1 were determined in AML cell lines (HEL, NB4, HL-60 and THP-1) and normal HS-5 cells. *P $<0.05$ vs HS- 5 cells or control group.
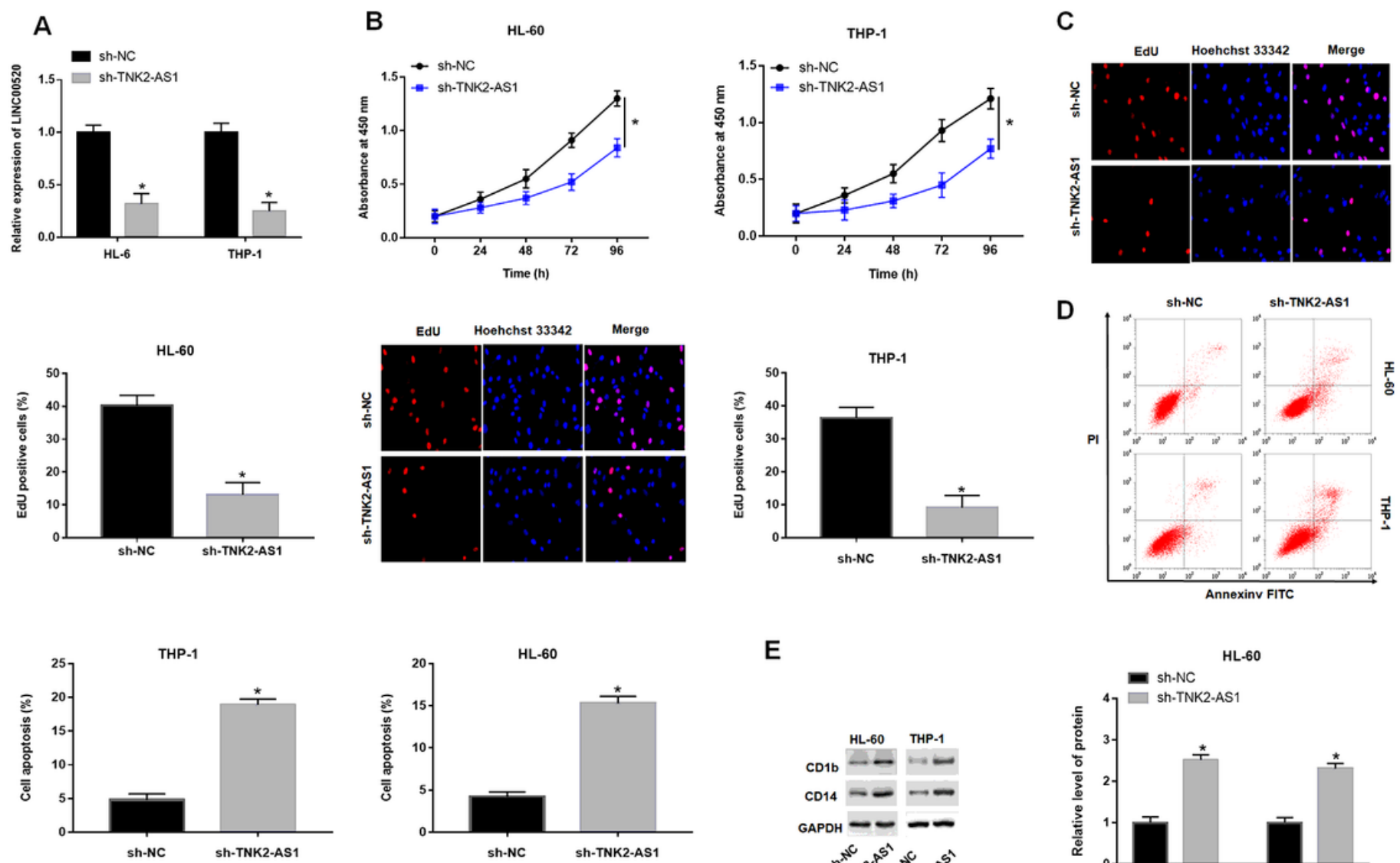

$E$
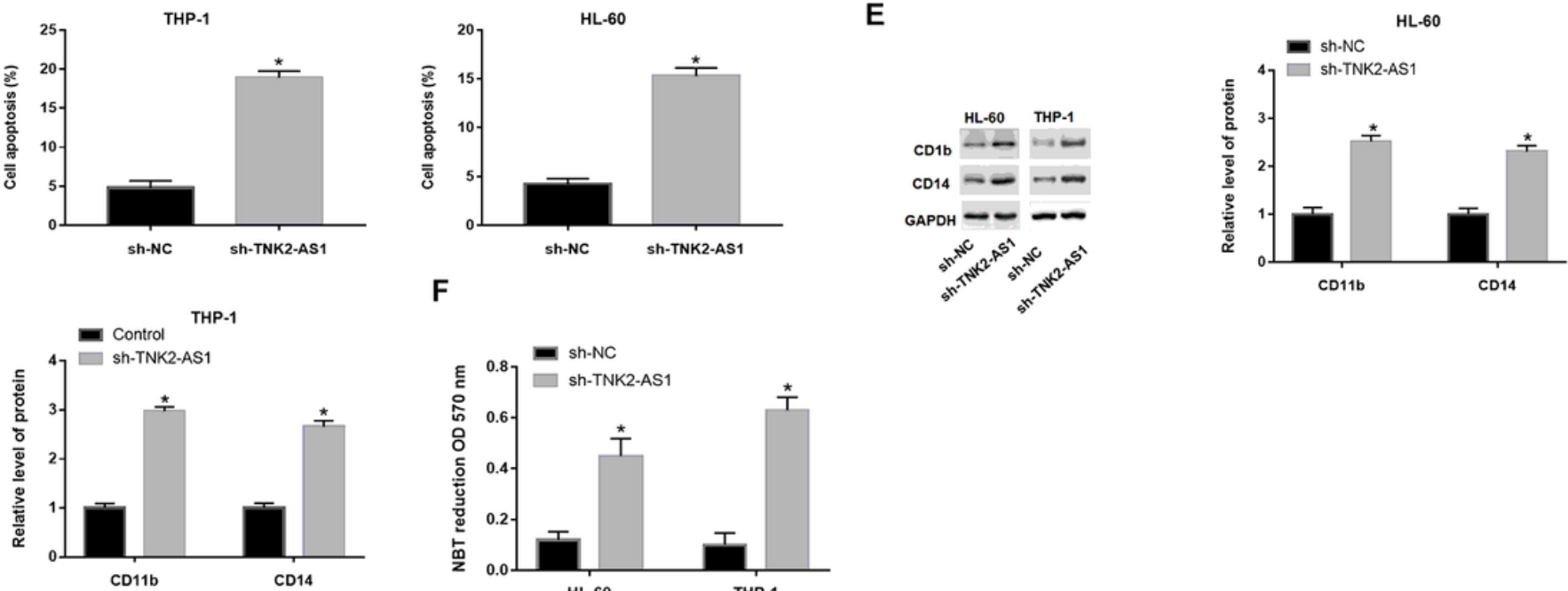

F
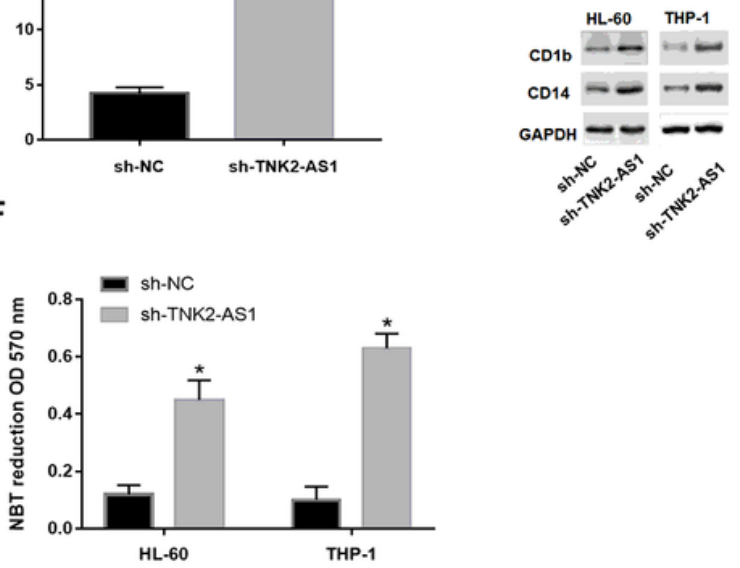

\section{Figure 2}

Knockdown of TNK2-AS1 inhibited AML cell growth and promoted differentiation. HL-60 and THP-1 cells were transfected with sh-NC and sh-TNK2-AS1. (A) The transfection efficiency was detected by RT-qPCR. (B-C) Cell proliferation of HL-60 and THP-1 cells was measured by CCK-8 and EdU assays. (D) Cell apoptosis of HL-60 and THP-1 cells was evaluated with flow cytometry. (E) The levels of CD11b and CD14 protein in HL-60 and THP-1 cells were assessed by western blotting assays. (F) The differentiation 
ability of HL-60 and THP-1 cells was detected by evaluating NBT reduction ability. *P $<0.05$ vs control group

A
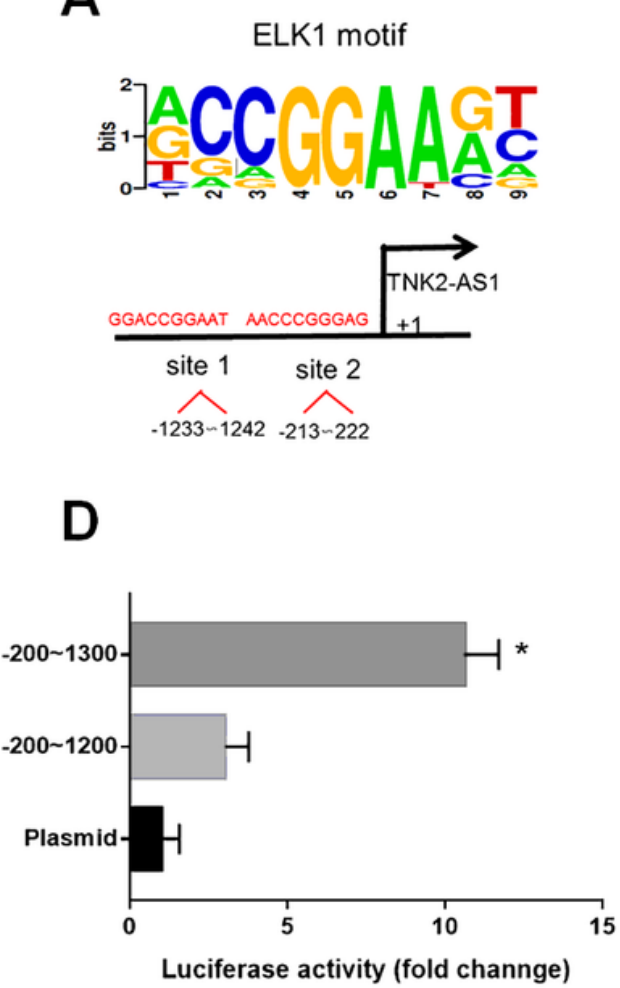

B
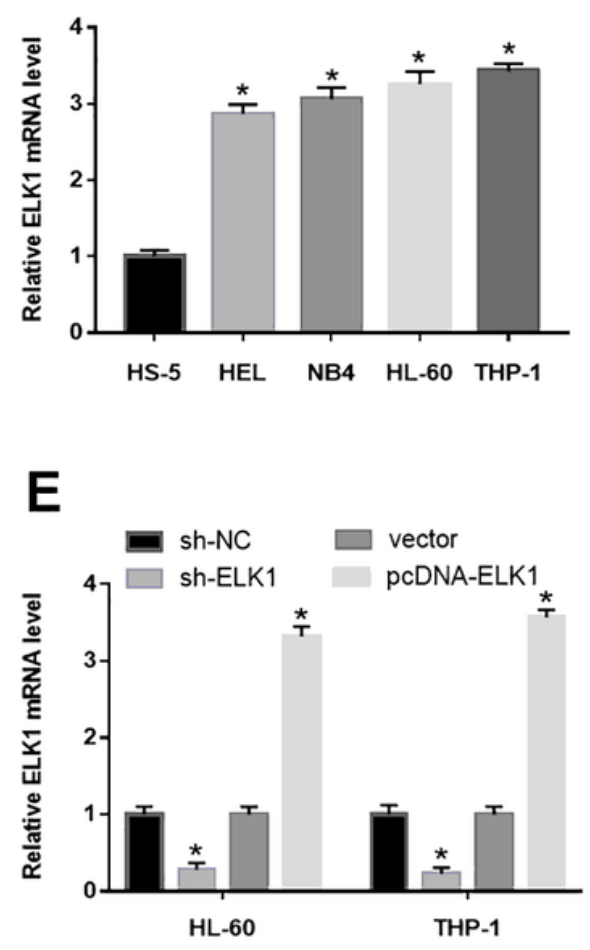

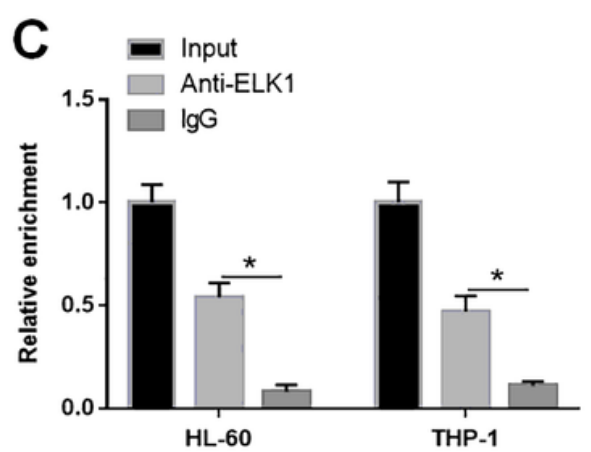

$\mathbf{F}$

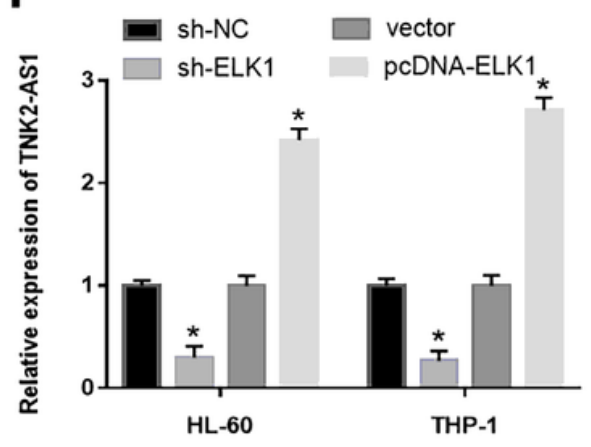

Figure 3

TNK2-AS1 expression was increased by the transcription factor ELK1 in AML cells. (A) The binding sites of ELK1 to TNK2-AS1 promoter was obtained from JASPAR. (B) The expression of ELK1 was determined in AML cell lines (HEL, NB4, HL-60 and THP-1) and normal HS-5 cells. (C) ChIP assay was performed in HL-60 and THP-1 cells to confirmed the binding of ELK1 to the promoter region of TNK2-AS1. (D) Luciferase reporter assay was utilized to measure the luciferase activity of three serial truncations in HEK293 T cell. (E) The regulation of ELK1 on the mRNA expression of TNK2-AS1 was demonstrated by RTqPCR. ${ }^{*} \mathrm{P}<0.05$ vs HS- 5 cells or IgG group or control group. 
A
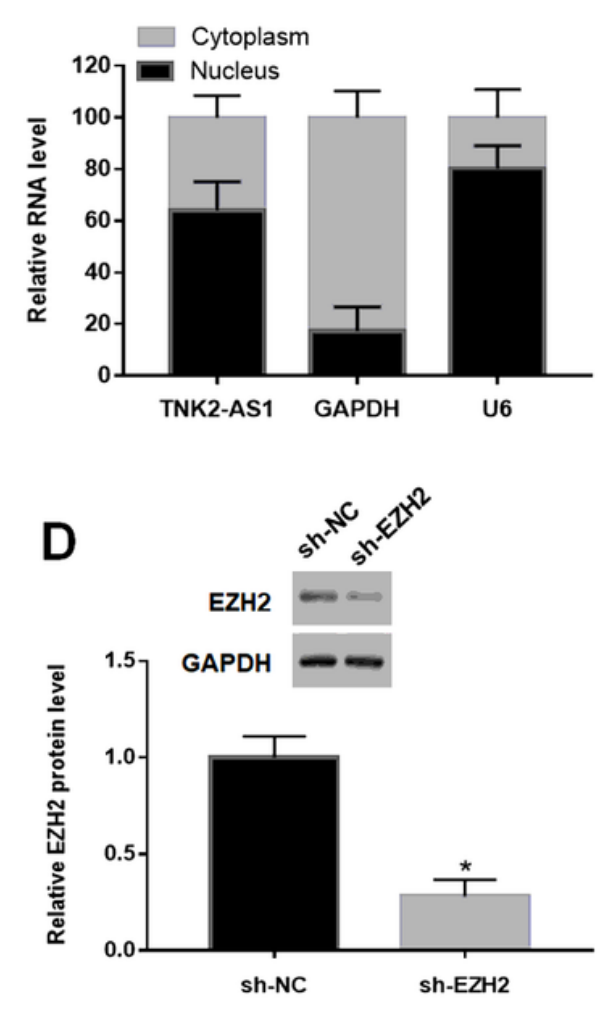

B
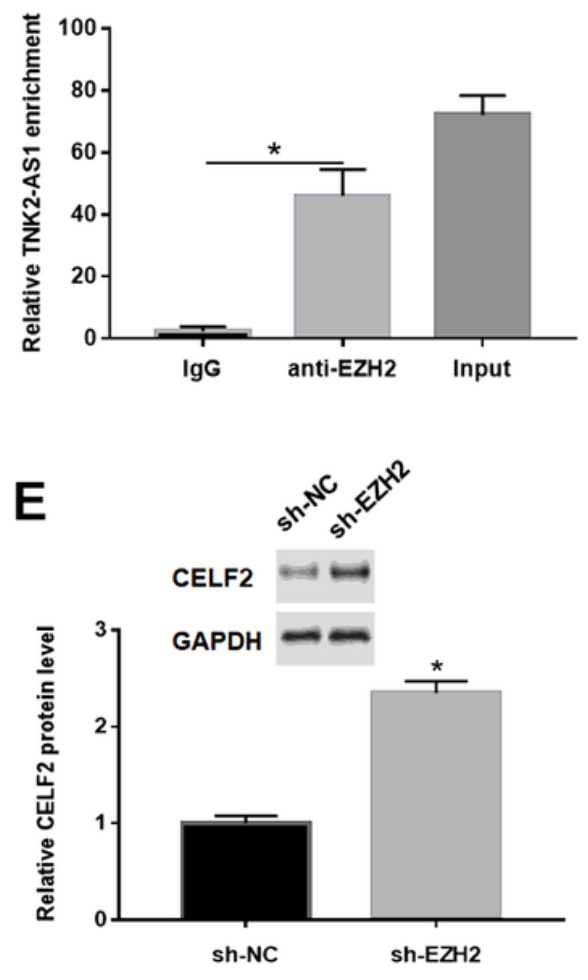

C

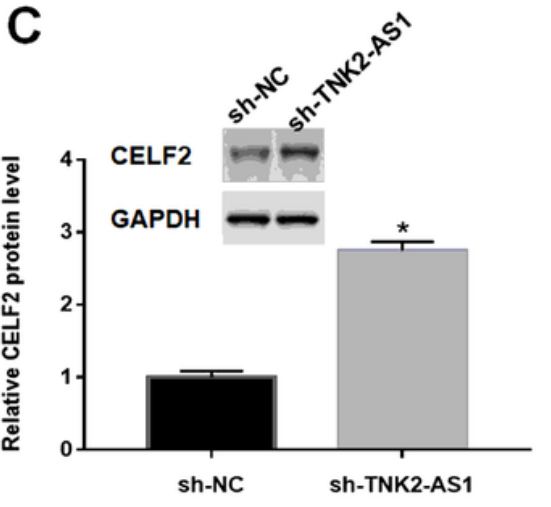

$\mathbf{F}$

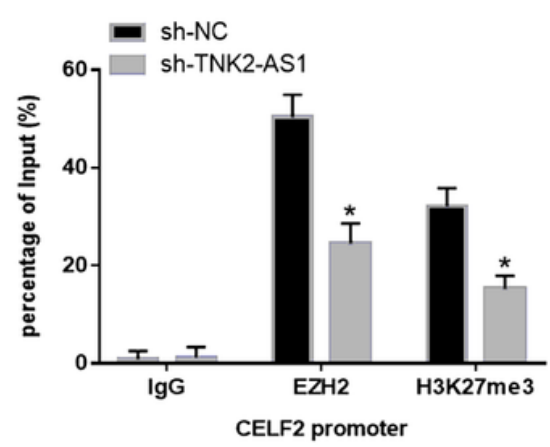

Figure 4

TNK2-AS1 regulated CELF2 expression by interacting with EZH2 in AML cells. (A) The nuclear location of TNK2-AS1 was determined in HL-60 cells. (B) RIP assay identified the interaction between TNK2-AS1 and EZH2 in HL-60 cells. (C) RT-qPCR and Western blotting analysis of CELF2 expression in HL-60 cells transfected with sh-NC and sh-TNK2-AS1 (D-E) RT-qPCR and Western blotting analysis of EZH2 expression in HL-60 cells transfected with sh-NC and sh-EZH2. (F) ChIP analysis of EZH2 occupancy and H3K27me3 binding in the CELF2 promoter in HL-60 cells transfected with sh-NC and sh-TNK2-AS1. *P < $0.05 \mathrm{vs}$ IgG group or control group. 
A
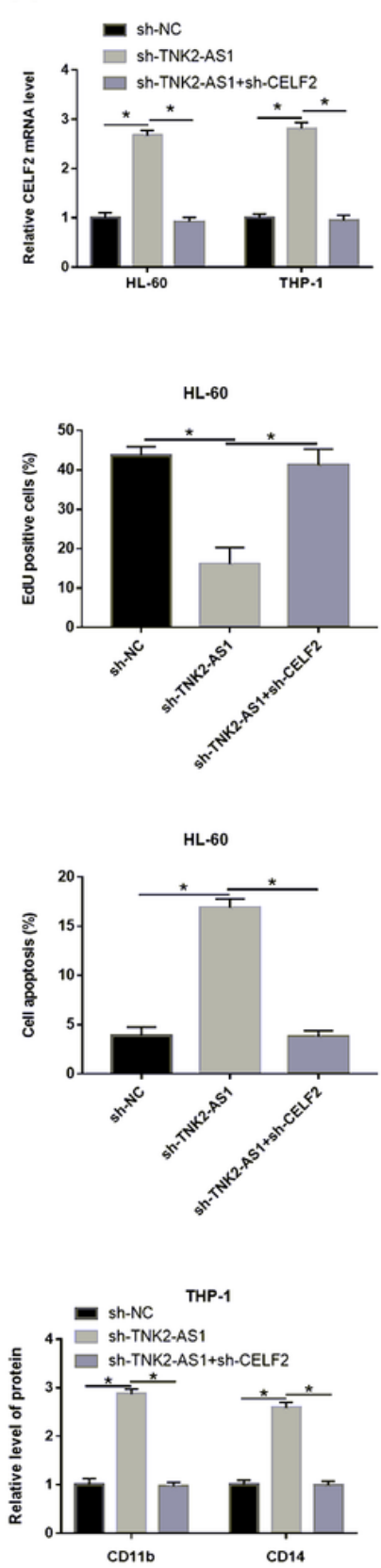

B
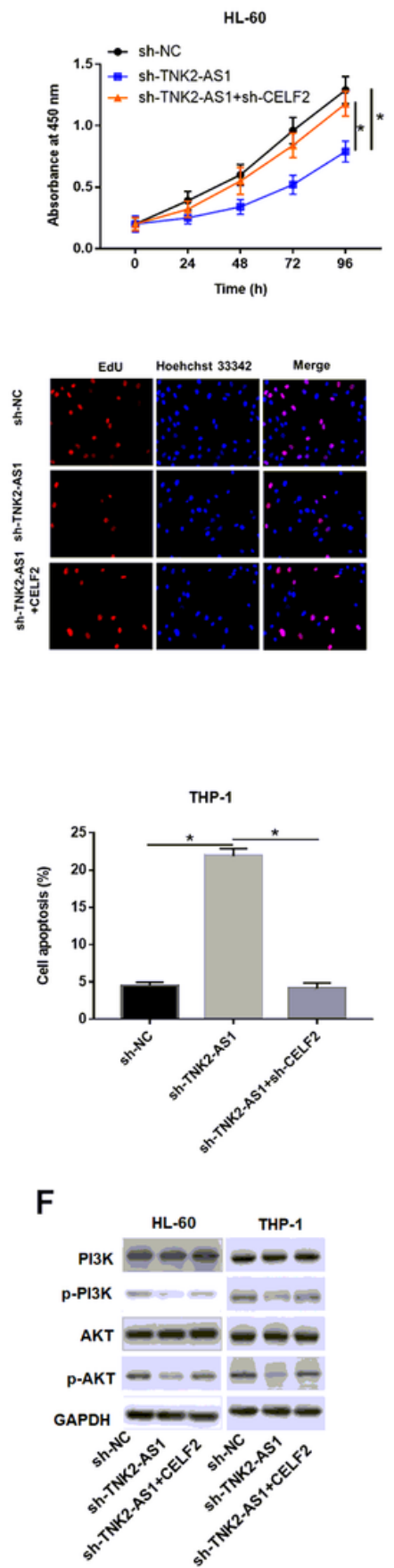

C
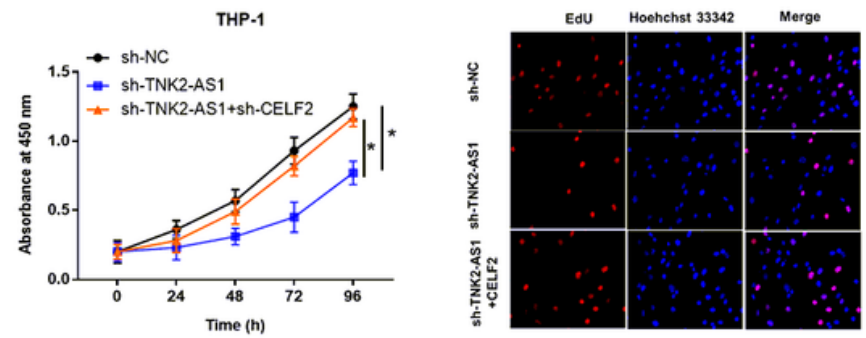

D
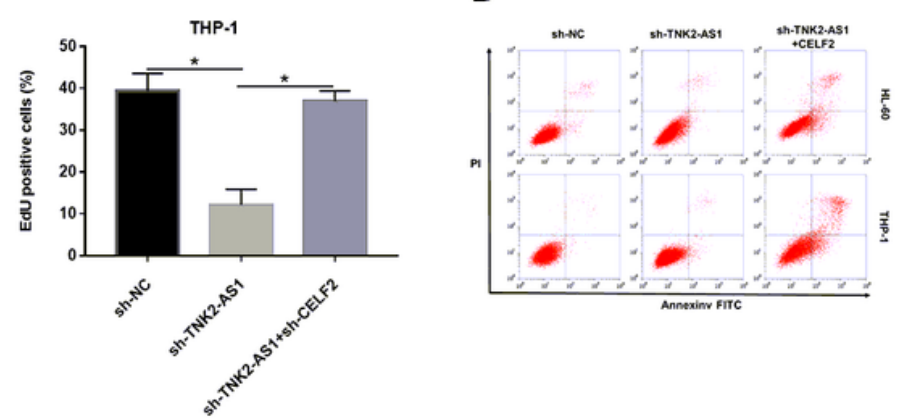

E
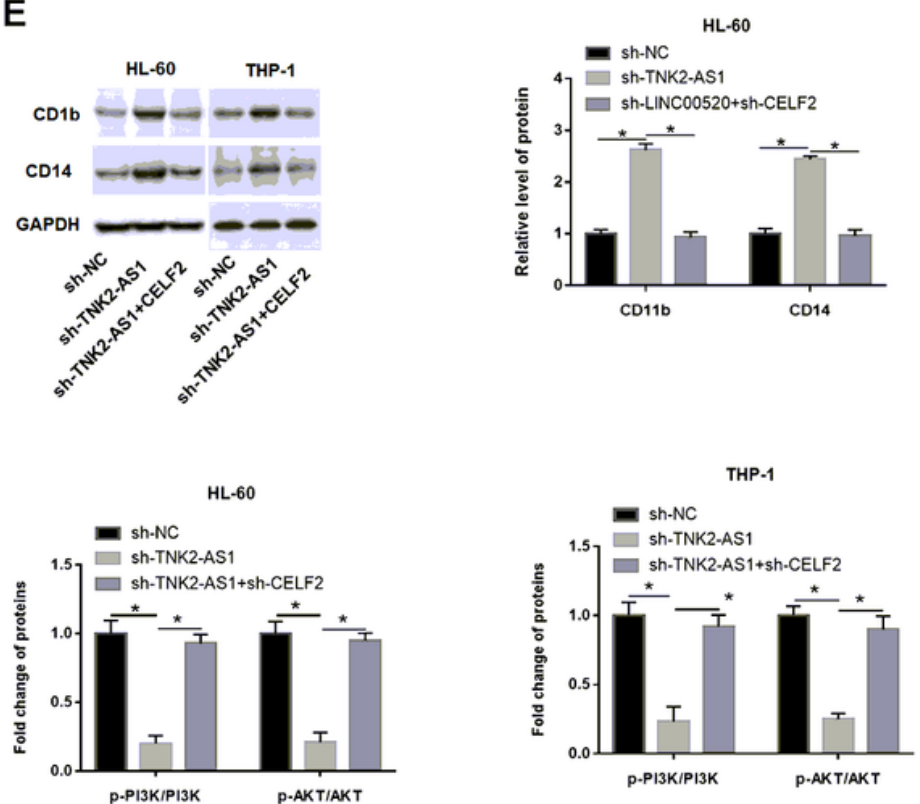

\section{Figure 5}

TNK2-AS1 exerted oncogenic effect by inhibiting the expression of CELF2. HL-60 and THP-1 cells were transfected with sh-TNK2-AS1 along or together with sh-CELF2. (A) The transfection efficiency was detected by RT-qPCR. (B-C) Cell proliferation of HL-60 and THP-1 cells was measured by CCK-8 and EdU assays. (D) Cell apoptosis of HL-60 and THP-1 cells was evaluated with flow cytometry. (E) The levels of CD11b and CD14 protein in HL-60 and THP-1 cells were assessed by western blotting assays. (F) The levels of p-PI3K and p-Akt expression in HL-60 and THP-1 cells were assessed by western blotting. *P < 0.05 vs control group or sh-TNK2-AS1. 
A
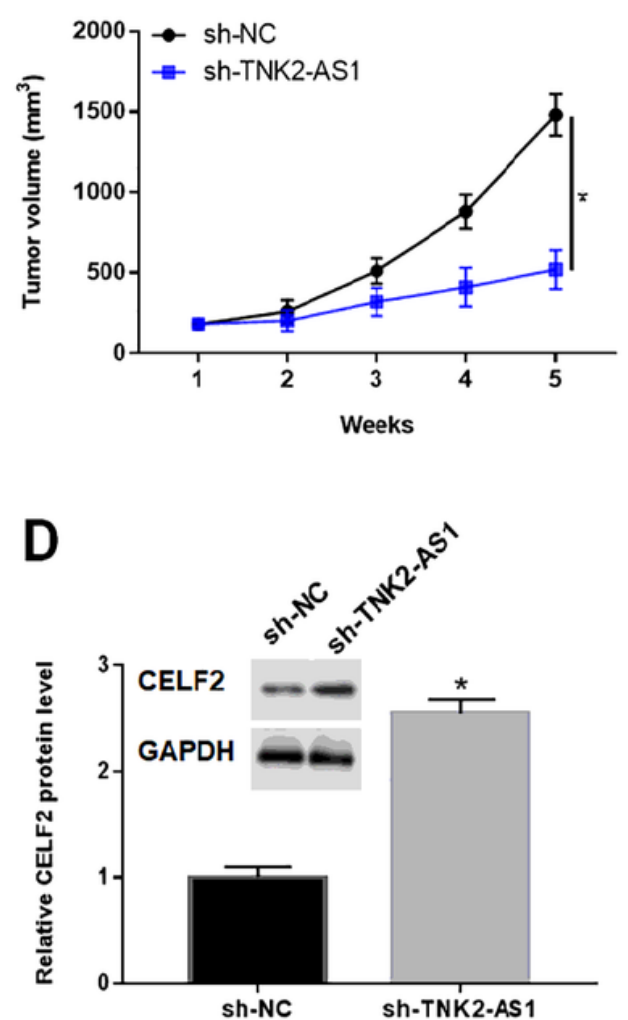

B

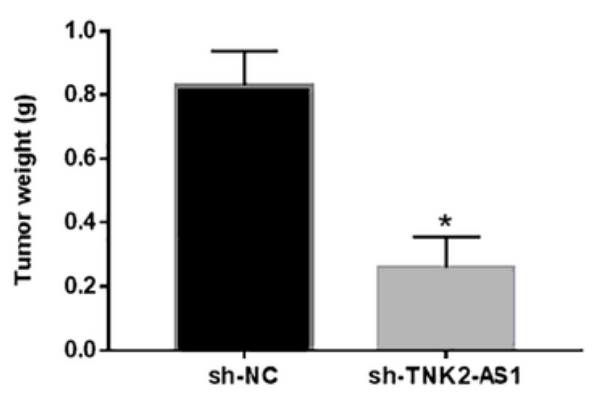

E

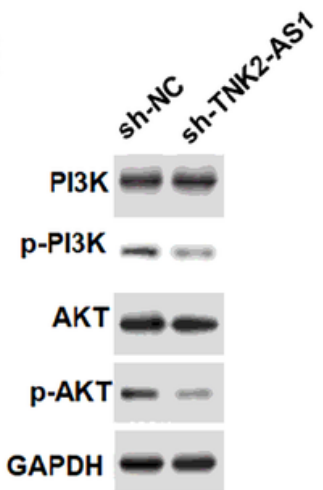

C
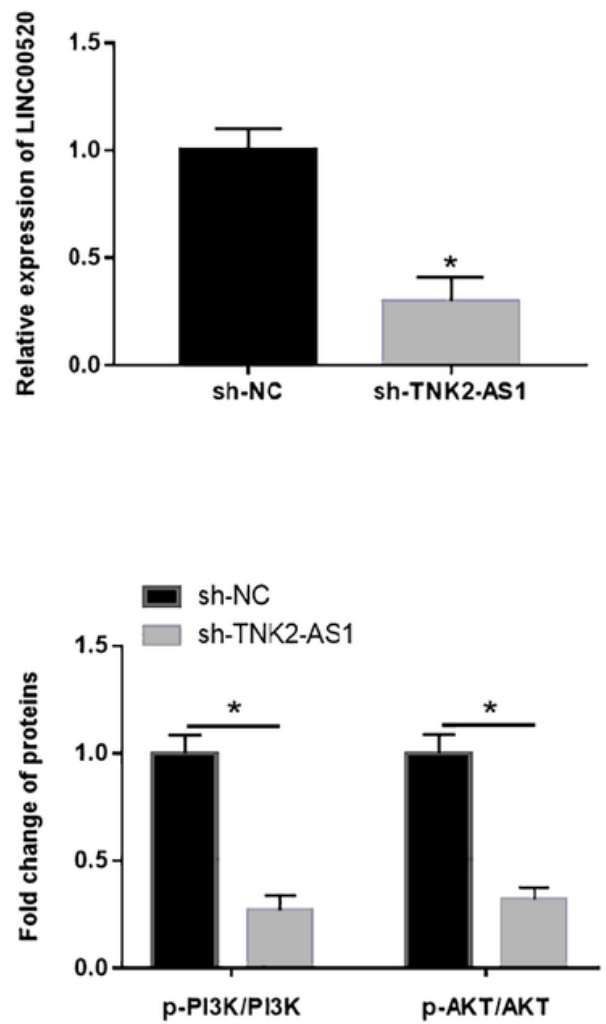

Figure 6

Silencing TNK2-AS1 suppressed AML tumorigenesis in vivo. AML cells stably transfected with sh-NC or sh-TNK2-AS1 were injected into nude mice (A) Tumor volume was calculated every week after inoculation. (B) Tumor weight was weighted in mice. (C-D) The expression of TNK2-AS1 and CELF2 was measured by RT-qPCR and Western blot. (E) The levels of p-PI3K and p-Akt expression in mice was detected by RT-qPCR and Western blot assay. ${ }^{*} \mathrm{P}<0.05$ vs control group. 